\title{
Fabrication of Electrospun Levodopa-Carbidopa Fixed-Dose Combinations
}

\author{
Haitham Bukhary ${ }^{1,2} \cdot$ Gareth R. Williams $^{1}$ (D) Mine Orlu ${ }^{1}$ \\ Received: 28 November 2019 / Accepted: 3 February 2020 / Published online: 2 March 2020 \\ (c) The Author(s) 2020
}

Keywords Electrospinning $\cdot$ Nanofiber $\cdot$ Fixed-dose combination $\cdot$ Polypharmacy

\section{Introduction}

Parkinson's disease (PD) is a disabling disorder that significantly affects a patient's quality of life [1]. Treatments are available, but only $10 \%$ of PD patients fully adhere to their treatment regimens [2]. Non-adherence is a serious problem linked to worsening symptoms and increased motor fluctuations [3-5]. Levodopa (LD) remains the most effective treatment for PD [6]. However, a single dosing treatment is only effective in the early phase of the disease. In the severe phase, 4-5 years after diagnosis, the therapeutic window of dopamine becomes narrow, and due to its short half-life of between 0.7 and $1.4 \mathrm{~h}$ dosing of LD will be required every $2 \mathrm{~h} \mathrm{[7-9].} \mathrm{One} \mathrm{way} \mathrm{to} \mathrm{ameliorate} \mathrm{this} \mathrm{issue} \mathrm{is} \mathrm{to} \mathrm{co-admin-}$ ister LD with carbidopa (CD), which can increase LD's oral bioavailability to $40-70 \%$ [10].

A major goal of PD pharmacotherapy has been the development of an oral formulation able to keep LD in the therapeutic plasma concentration range [11]. The timely intake of LD is of crucial importance to avoid complications arising [12]. There exists an approved combination formulation of LD and CD, available in a dose range between 100/25 and 200/50 mg (Sinemet $\mathrm{CR}^{\circledR}$; Merck). This takes the form of sustained-release (SR) tablets which release their ingredients over a 4 to $6 \mathrm{~h}$ period $[11,13]$. The SR tablets are advantageous over an immediate release (IR) formulation of LD alone, because SR can reduce fluctuations of dose in the

Gareth R. Williams

g.williams@ucl.ac.uk

$\triangle$ Mine Orlu

m.orlu@ucl.ac.uk

1 UCL School of Pharmacy, University College London, 29-39 Brunswick Square, London WC1N 1AX, UK

2 Department of Pharmaceutics, College of Pharmacy, Umm Al-Qura University, Makkah 24381, Saudi Arabia systemic circulation [5]. However, LD absorption might be delayed if the SR tablet is used alone, so effective therapy often requires co-administration of an IR dose of LD/CD, particularly for the first dose of the day [11]. In this work, we seek to combine both immediate and sustained release of LD and CD into a single formulation, with the ultimate aim of improving patient compliance in PD treatment.

The formulation approach taken here is to use electrospinning to produce drug-loaded polymer fibers. Electrospinning first involves the preparation of a mixed solution of a polymer and drug in a volatile solvent, followed by ejection of this solution through a narrow-bore needle and the application of an electrical field to solidify the product. It is beneficial over other techniques such as spray drying and hot-melt extrusion because it does not require the application of heat, and thus thermally labile active ingredients can be processed [14]. Further, electrospinning allows multiple different fluids to be processed simultaneously, leading to fiber products with highly tunable properties $[15,16]$. Electrospun fibers have been widely explored to generate drug delivery systems, and there are reports in the literature of fast-dissolving [17-20], extended release [21-23], and $\mathrm{pH}$ responsive systems [24, 25], among many others. Fixed-dose combination formulations have also been prepared by electrospinning [26, 27], but there are few studies where multiple drugs have been combined into a single fiber formulation.

The most simple electrospinning experiment involves a single polymer/drug solution, and results in a monolithic product with the drug uniformly dispersed throughout the carrier in most cases. Coaxial electrospinning, dispensing two fluids simultaneously to prepare core/shell fibers, is a more complex process but can create formulations with a number of benefits over monolithic systems from singleneedle electrospinning [28, 29]. For instance, while a burst of drug release is typically observed from monolithic fibers (because of the presence of drug at the surface), this 
can be avoided in a core/shell system by having a drugfree shell layer [30]. Obviating this burst release is particularly important for hydrophilic small molecule drugs [31]. Other benefits of coaxial spinning lie in the development of drug delivery systems with multi-phasic release, as is desired here. Because coaxial electrospinning allows the combination of two polymers with very different properties (e.g. in terms of solubility/dissolution rate), it can result in products giving release at two different rates. This has been shown to be the case when polyvinylpyrrolidone (PVP) and zein were used to construct core/shell fibers [32].

In this work, for the first time, coaxial electrospinning was used to generate FDCs in the form of core/shell fibers (see Fig. 1a) designed for the treatment of Parkinson's disease. The FDC formulations are prepared using two base polymer solutions containing PVP [shell] and Eudragit ${ }^{\circledR}$ RL PO (E-RLPO) [core] (Fig. 1b). The aim of the work is to fabricate oral FDCs giving an initial burst release to provide a loading dose of the drug, followed by extended release. An immediate burst of drug release from the PVP shell is expected, since PVP is a well-known hydrophilic polymer and will dissolve rapidly upon contact with gastric fluids. This should be followed by extended release from the E-RLPO core, since the latter is insoluble in water but undergoes $\mathrm{pH}$-independent swelling in the digestive fluid to provide time-controlled release. The FDC formulation parameters were first optimised, before two sets of fibers were produced. These were characterised in detail using electron microscopy, $\mathrm{X}$-ray diffraction, differential scanning calorimetry, and IR spectroscopy. The drug loading and encapsulation efficiency were calculated, and the fibers' drug release profiles explored.

\section{Experimental Section}

\section{Materials}

Levodopa (LD), carbidopa (CD), and PVP (MW 360,000 Da) were purchased from Sigma-Aldrich, UK. Eudragit ${ }^{\circledR}$ RLPO (E-RLPO; MW 150,000 Da) was a gift from Evonik (Germany). Analytical grade ethanol, acetonitrile, methanol, and N,N-dimethylacetamide (DMAc) were procured from Sigma-Aldrich (UK). Deionised water was used where needed, and all other chemicals used were of analytical grade.

\section{Solution Preparation}

Two base polymer solutions prepared in ethanol were used in this work. These comprised 10\% w/v PVP and 20\% w/v Eudragit RLPO (E-RLPO). Each solution was stirred overnight to ensure complete dissolution. The required volume of each polymer solution was then mixed with a solution of the active pharmaceutical ingredients (APIs) dissolved in $1 \mathrm{M}$ aqueous $\mathrm{HCl}$. Full details of the formulations are given in Table 1. In order to protect LD and CD from photolytic degradation, the samples were stored in dark amber glass containers. The combined drug/polymer solutions were mixed at room temperature for $6 \mathrm{~h}$ to obtain homogeneous solutions.
Fig. 1 A schematic diagram of the coaxial electrospinning process showing a the experimental setup and $\mathbf{b}$ the core/shell FDCs prepared in this work (a)

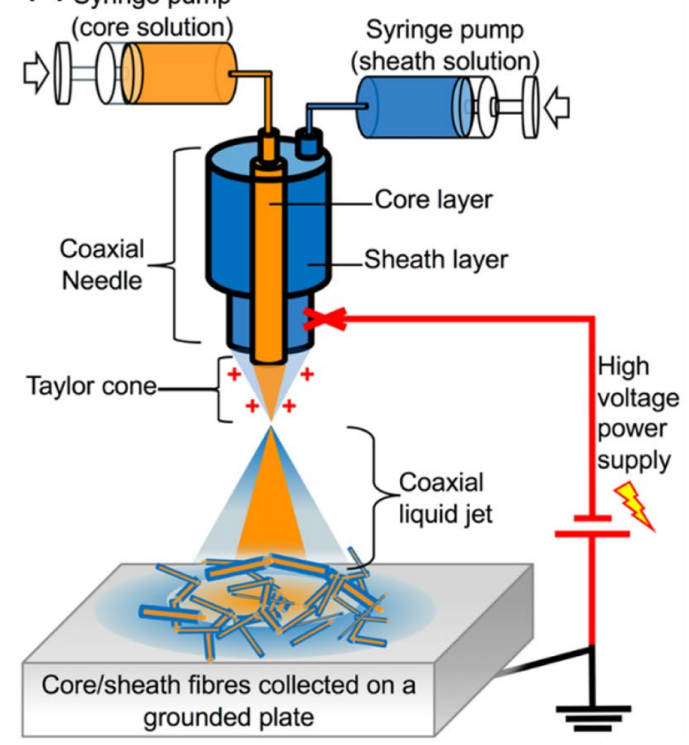

(b)

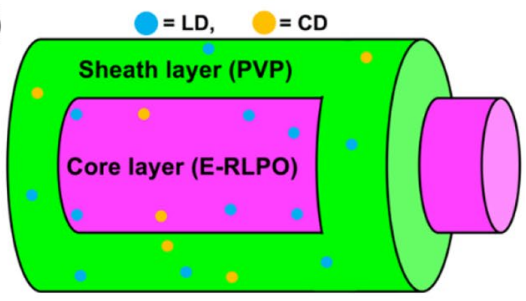


Table 1 The compositions of each formulation, and the processing parameters explored

\begin{tabular}{lll}
\hline & \multicolumn{2}{l}{ Formulation } \\
\cline { 2 - 3 } & F1 & F2 \\
\hline Theoretical LD content in fibers (\% w/w) & 19.0 & 8.9 \\
Theoretical CD content in fibers (\% w/w) & 4.8 & 2.2 \\
Core solution composition & & \\
Volume of E-RLPO solution (mL) & 2 & 5 \\
Volume of LD/CD solution (mL) & 1 & 1 \\
Concentration of LD/CD solution (mg/mL) & $100 / 25$ & $100 / 25$ \\
Shell solution composition & & \\
Volume of PVP shell solution (mL) & 4 & 10 \\
Volume of LD/CD solution (mL) & 2 & 2 \\
Concentration of LD/CD solution (mg/mL) & $50 / 12.5$ & $50 / 12.5$ \\
Electrospinning parameter optimisation & & \\
Range of flow rates explored (mL/h) & & \\
Core & $0.2-0.6$ & \\
Shell & $0.6-1.2$ & \\
Range of voltages explored (kV) & $18-24$ & \\
Range of spinneret to collector distances (cm) & $13-19$ & \\
Optimised electrospinning parameters & & \\
Flow rate (mL/h) & & \\
Core & & \\
Shell & & \\
Voltage (kV) & & \\
Spinneret to collector distance (cm) & 1.2 & \\
\hline
\end{tabular}

\section{Electrospinning}

The setup of the coaxial electrospinning apparatus is shown in Fig. 1a. For each formulation, the polymer solutions were loaded into separate plastic syringes (BD plastic, MediSupplies, UK) with care taken to avoid any air bubbles. Each syringe was fitted on a syringe pump (KDS100, Cole Parmer, UK) and connected with plastic tubing to infuse the polymer solution into a concentric spinneret comprising two nested stainless steel needles (core: 18G, $0.83 \mathrm{~mm}$ inner diameter; shell: $13 \mathrm{G}, 1.83 \mathrm{~mm}$ inner diameter). A positive voltage was applied to the spinneret using an HCP 35-35000 power supply (FuG Elektronik GmbH, Germany). To collect the fibers, a flat metal collector $(5 \times 5) \mathrm{cm}$ covered with aluminium foil was situated below the spinneret tip. The electrospinning process took place in ambient conditions of 19 to $26^{\circ} \mathrm{C}$, and 31 to $49 \%$ relative humidity.

Optimisation of the electrospinning parameters was first performed to find the most appropriate flow rate, voltage, and spinneret to collector distance. The range of parameters explored and optimal processing conditions identified are detailed in Table 1.

\section{Characterisation}

Scanning electron microscopy (SEM) images were obtained on a Quanta 200 FEG SEM (FEI, USA). Prior to examination, samples were sputter-coated with gold under argon to make them electrically conductive. Images were taken at an excitation voltage of $5 \mathrm{kV}$. The average diameter of the fiber formulations was determined from the SEM images by making manual measurements at 100 different points using the ImageJ software (National Institutes of Health, USA).

The electrospun fibers were also examined by transmission electron microscopy (TEM). For this, samples were electrospun directly onto a lacy carbon coated copper grid (TAAB, UK). TEM images were taken on a Philips/FEI CM 120 Bio-Twin instrument (Netherlands).

$\mathrm{X}$-ray diffraction (XRD) patterns were obtained on a MiniFlex 600 diffractometer (Rigaku, Japan) supplied with $\mathrm{Cu} \mathrm{K} \alpha$ radiation $(\lambda=1.5418 \AA)$ at a voltage of $40 \mathrm{kV}$ and current of $15 \mathrm{~mA}$. Samples were fixed on an aluminium holder and data recorded over the $2 \theta$ range between $3^{\circ}$ and $43^{\circ}$ at a scan speed of $5^{\circ} \mathrm{min}^{-1}$. Differential scanning calorimetry (DSC) studies were conducted using a Q2000 calorimeter (TA Instruments, USA). Non-hermetically sealed samples in aluminium pans (Tzero premium pan/ lid, TA instruments) were heated from $30^{\circ} \mathrm{C}$ to an appropriate temperature (ideally above the melting point of the active ingredient of interest but below the degradation point of the polymer). Experiments were undertaken at a rate of $10^{\circ} \mathrm{C} \mathrm{min}{ }^{-1}$ and under a nitrogen flow of $50 \mathrm{~mL} \mathrm{~min}^{-1}$. Data were analysed using the TA instruments Universal Analysis software.

Samples from each formulation were analysed in attenuated total reflectance (ATR) mode IR spectroscopy on a Spectrum 100 instrument (PerkinElmer, USA). Samples were studied over the range 4000 to $650 \mathrm{~cm}^{-1}$, with the spectral resolution set at $1 \mathrm{~cm}^{-1}$. Four scans per sample were recorded.

\section{Functional Performance Assessment}

A high performance liquid chromatography (HPLC) assay for simultaneous analysis of both $\mathrm{LD}$ and $\mathrm{CD}$ was devised based on the method for Co-Careldopa detailed in the British Pharmacopeia [33]. The analysis was performed on a 1200 Infinity Series instrument (Agilent Technologies, USA). The stationary phase was a C18(2) column (00G-4252-E0, Phenomenex Luna, UK) held at $25^{\circ} \mathrm{C}$. Analysis was carried out at a flow rate of $1.5 \mathrm{~mL} \mathrm{~min}^{-1}$, and an injection volume of $20 \mu \mathrm{L}$ was used. A UV detector was used for quantification at $282 \mathrm{~nm}$, where LD can be detected at $\mathrm{t}=3.7 \mathrm{~min}$ and $\mathrm{CD}$ at $\mathrm{t}=6.2 \mathrm{~min}$. The mobile phase comprised 20:80 v/v methanol: phosphate buffered saline (PBS; pH 2.8, 
$0.01 \mathrm{~mol} / \mathrm{L}$ ). Adjustment of the PBS $\mathrm{pH}$ was undertaken using $1 \mathrm{M}$ orthophosphoric acid (85\%, HPLC grade, Fisher Scientific, UK). The mobile phase solutions were mixed and filtered through a $0.22 \mu \mathrm{m}$ membrane filter (Millex syringe filter, Merck Millipore, Germany) before use.

The loading of each drug in the electrospun fibers was evaluated by taking $20 \pm 1 \mathrm{mg}$ of each sample and dissolving this in $100 \mathrm{~mL}$ of $1 \mathrm{M} \mathrm{HCl}$ under stirring. The vessels used all comprised darkened glass to protect the samples from light degradation. A calibration curve for $\mathrm{LD}$ and $\mathrm{CD}$ was prepared from stock solutions stored in the dark and freshly diluted on the day of the experiment.

Drug release experiments were carried out using USP apparatus type 1, with a rotating basket (ST7 dissolution tester, Caleva, UK). Fiber samples of $30 \pm 1 \mathrm{mg}$ were loaded in hard gelatin capsules (size 0). Each capsule was placed in the rotating basket at $50 \mathrm{rpm}$, then immersed in a vessel containing $750 \mathrm{~mL}$ of $0.1 \mathrm{M} \mathrm{HCl}$, at a temperature of $37{ }^{\circ} \mathrm{C}$. At predetermined time points, $1 \mathrm{~mL}$ samples were withdrawn and replaced with an equal amount of fresh preheated medium. The release of each API was quantified by HPLC as described above. Three independent experiments were carried out, and cumulative release percentages are reported as mean \pm S.D.

\section{Results and Discussion}

\section{Fiber Morphology}

SEM images of the raw APIs and the coaxial electrospun fibers $\mathrm{F} 1$ and F2, together with the diameter distributions of the latter, are displayed in Fig. 2. The SEM images of the raw materials (Fig. 2) show LD to exist as large particles with sharp edges and CD as elongated particles with irregular edges. This is consistent with both being crystalline materials. The production of fibers was successful for both formulations, as shown in Fig. 2. The images of the fibers do not show any evidence for particles of LD or $\mathrm{CD}$, indicating a solid dispersion was generated. In general the fibers have cylindrical shapes with a smooth surfaces, although for F2 some flattened fibers can also be observed. The fiber diameters are $380 \pm 120 \mathrm{~nm}$ and $1300 \pm 330 \mathrm{~nm}$ for $\mathrm{F} 1$ and $\mathrm{F} 2$ respectively. This difference can be attributed to the greater amount of solid material dispensed per unit time with F2. Similar results have been reported by Pornsopone et al. [34, 35], who produced fibers sized $\sim 1300 \mathrm{~nm}$ from $20 \% \mathrm{w} / \mathrm{v}$ E-RLPO, and fibers sized $2200 \mathrm{~nm}$ from $25 \%$ w/v E-RLPO.

TEM images of the fibers are shown in Fig. 3. Samples were collected at different times after electrospinning was commenced, in order to investigate whether core/shell fibers were continually produced. The images of F1 (Fig. 3a, b) reveal the formation of core/shell fibers in both samples collected, with the core around 154-160 nm in diameter and the shell at 226-295 nm. The images of F2 (Fig. 3c, d) do not confirm the formation of core/shell fibers, however. It appears that here monolithic fibers have been produced. It is not clear why this is, but the solutions used for F2 fabrication have higher polymer concentrations than those used for F1. This will result in them having higher viscosities and different drawing and drying behaviour under the electric field. As a result of these factors, it appears that mixing of the fluids occurred during spinning.

\section{Physical Form and Component Compatibility}

The physical form of the drugs in the fibers was assessed with XRD and DSC. The XRD patterns are given in Fig. 4. The patterns of the API raw materials show numerous sharp Bragg reflections for $\mathrm{LD}$ (e.g. at $18.36^{\circ}, 21.40^{\circ}, 22.98^{\circ}$, and $25.08^{\circ}$ ) and $\mathrm{CD}\left(\right.$ e.g. at $9.66^{\circ}, 18.66^{\circ}$, and $19.56^{\circ}$ ). This illustrates that both drugs existed in the crystalline form before electrospinning. In contrast, the XRD patterns for the polymers show broad haloes without any Bragg reflections, which indicates the amorphous nature of both polymers. In the patterns of the fiber composites, only a broad halo is seen, without the presence of any characteristic reflections from $\mathrm{LD}$ or $\mathrm{CD}$. This confirmed that both drugs are present as amorphous solid dispersions within the fibers. This result was expected since it is known that electrospinning involves a very rapid drying process, which does not allow sufficient time for recrystallisation. Coaxial fibers prepared from PVP and other Eudragit polymers have been found to have similar amorphous structures [36, 37].

The DSC data for the raw API materials (Fig. 5) show endothermic peaks corresponding to melting, with onset for $\mathrm{LD}$ at $284{ }^{\circ} \mathrm{C}$ and for $\mathrm{CD}$ at $196{ }^{\circ} \mathrm{C}$. These results are consistent with values reported in the literature for $\mathrm{LD}$ (284-286 ${ }^{\circ} \mathrm{C}$; [38]) and $\mathrm{CD}\left(203-208{ }^{\circ} \mathrm{C}\right.$; [33, 39]). This observation indicates that both drugs were initially in the crystalline form before processing, and agrees with the findings from XRD. In contrast, the data for the polymer raw materials (Fig. 5) show no melting events, consistent with the XRD patterns and confirming them to be amorphous materials. Instead, PVP shows a broad endothermic peak at around 30 to $150{ }^{\circ} \mathrm{C}$ due to the evaporation of adsorbed water, and $\mathrm{T}_{\mathrm{g}}$ appears at $176^{\circ} \mathrm{C}$, close to the literature value of $166^{\circ} \mathrm{C}$ [40]. E-RLPO shows a $\mathrm{T}_{\mathrm{g}}$ at $53{ }^{\circ} \mathrm{C}$, followed by a broad dehydration endotherm between 67 and $140{ }^{\circ} \mathrm{C}$. The $\mathrm{T}_{\mathrm{g}}$ here is similar to a value previously reported for E-RLPO [41] $\left(53.1{ }^{\circ} \mathrm{C}\right)$. The events observed at $166{ }^{\circ} \mathrm{C}$ for E-RLPO have been described by Parikh et al. as polymer degradation [42].

The thermograms of the coaxial fibers (Fig. 5) show they undergo dehydration, in the form of a broad endotherm 
Fig. 2 SEM images of $\mathrm{LD}, \mathrm{CD}$, F1 (19.0/4.8\% w/w LD/CD), and F2 (8.9/2.2\% w/w LD/CD), with fiber diameter distributions
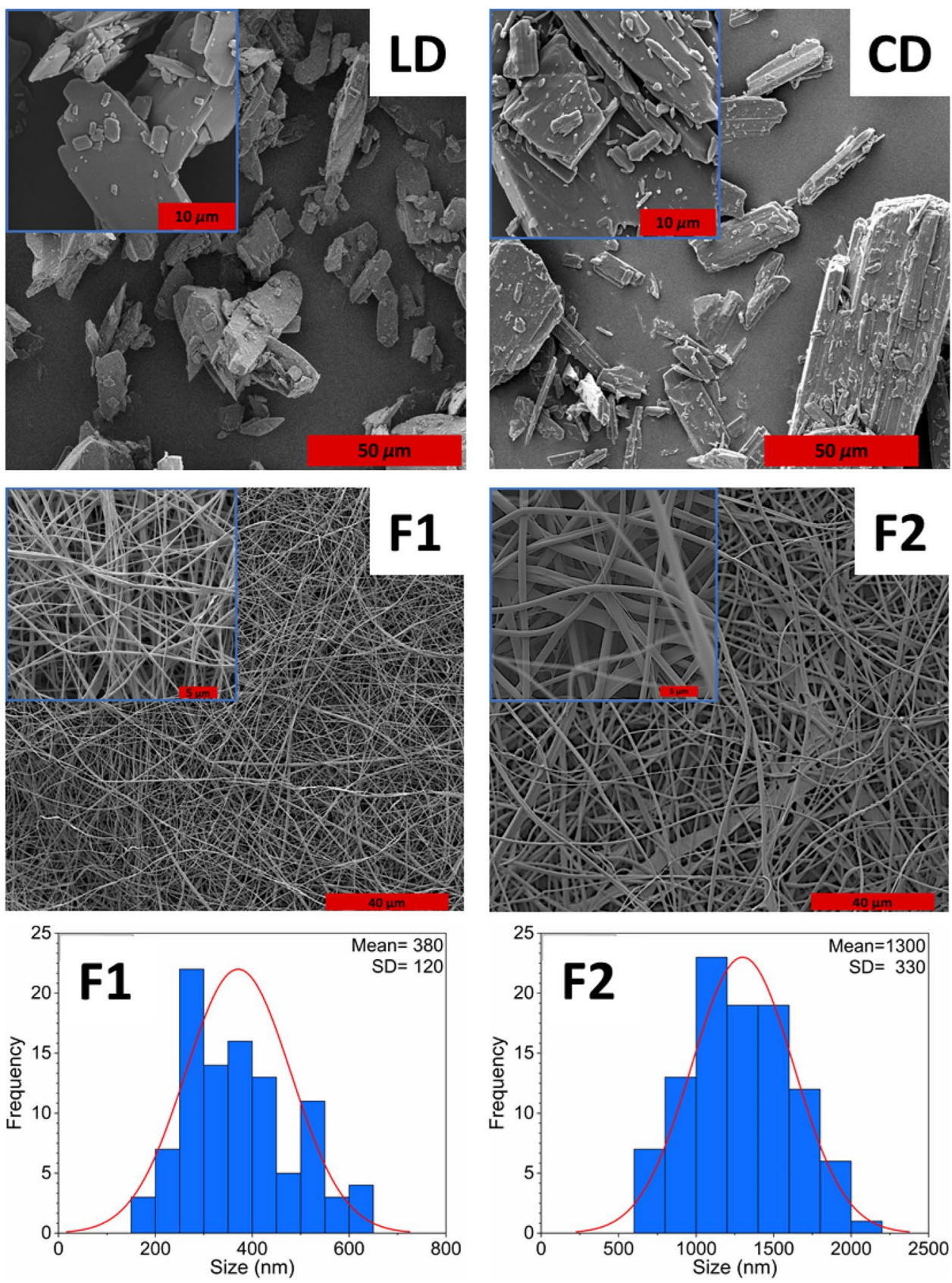

between room temperature and $140{ }^{\circ} \mathrm{C}$ for $\mathrm{F} 1$ or $150{ }^{\circ} \mathrm{C}$ for $\mathrm{F} 2$. These endotherms are very shallow, indicating minimal solvent inclusion in the systems. No melting endotherms from either $\mathrm{LD}$ or $\mathrm{CD}$ are visible for the fibers, which agrees with the findings from XRD and demonstrates the fibers to comprise amorphous solid dispersions. The thermogram of $\mathrm{F} 2$ shows $\mathrm{T}_{\mathrm{g}} \mathrm{s}$ at $56{ }^{\circ} \mathrm{C}$ from E-RLPO, and at $179{ }^{\circ} \mathrm{C}$ from PVP. In F1 these glass transitions cannot be clearly observed, but the noise at ca. $166^{\circ} \mathrm{C}$ could be related to E-RLPO degradation.

The chemical structures of the raw materials are presented in Fig. 6a. The FTIR spectra of the API raw materials (Fig. 6b) present characteristic bands for each material. In the spectrum of $\mathrm{LD}$, characteristic peaks appear from O-H stretching at 3200,3063, and $2982 \mathrm{~cm}^{-1}$, from phenyl group $\mathrm{C}=\mathrm{C}$ vibrations at $1458,1404,1352$, 817 , and $674 \mathrm{~cm}^{-1}$, and from $\mathrm{C}-\mathrm{H}$ stretches at 2929 and $2982 \mathrm{~cm}^{-1} . \mathrm{NH}_{2}$ stretches are visible at $1562 \mathrm{~cm}^{-1}$, and $\mathrm{C}=\mathrm{O}$ stretching at $1651 \mathrm{~cm}^{-1}$. In the spectrum of $\mathrm{CD}$, $\mathrm{O}-\mathrm{H}$ stretching bands arise at $3519,3322,3286 \mathrm{~cm}^{-1}$, phenyl group $\mathrm{C}=\mathrm{C}$ vibrations are present at 1457,1402 , 1262,879 , and $830 \mathrm{~cm}^{-1}$, and $\mathrm{C}-\mathrm{H}$ stretches can be seen at 3101,3059 , and $1371 \mathrm{~cm}^{-1} . \mathrm{N}-\mathrm{H}$ and $\mathrm{NH}_{2}$ stretches arise at $1527 \mathrm{~cm}^{-1}$ and $1122 \mathrm{~cm}^{-1}$, and $\mathrm{C}=\mathrm{O}$ stretching 


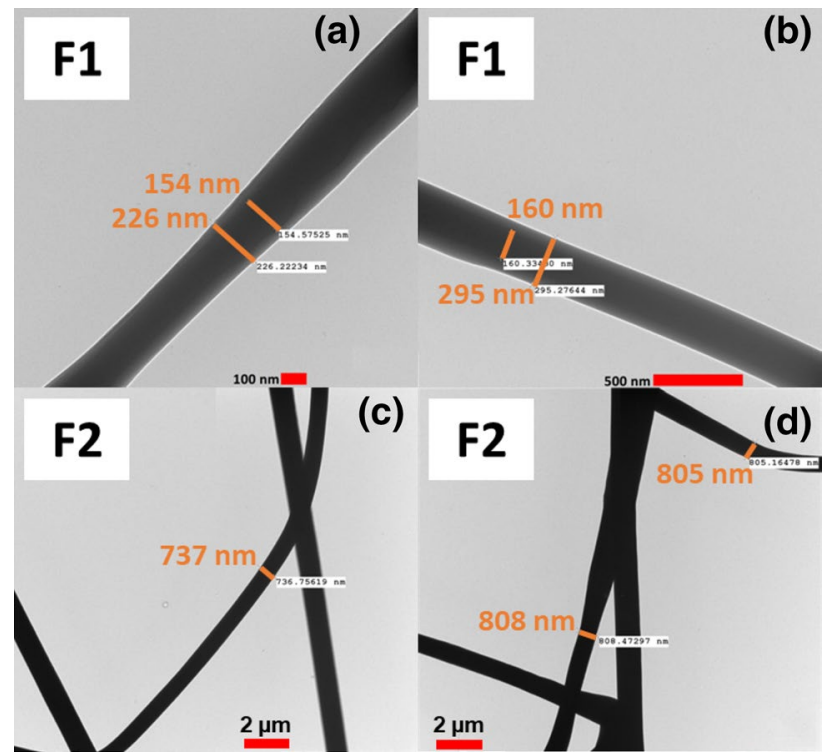

Fig. 3 TEM images of the fibers, showing $\mathrm{F} 1$ after dispensing a $3 \mathrm{~mL}$ and $\mathbf{b} 6 \mathrm{~mL}$ of liquid, and $\mathrm{F} 2$ after dispensing $\mathbf{c} 6 \mathrm{~mL}$ and $\mathbf{d} 12 \mathrm{~mL}$

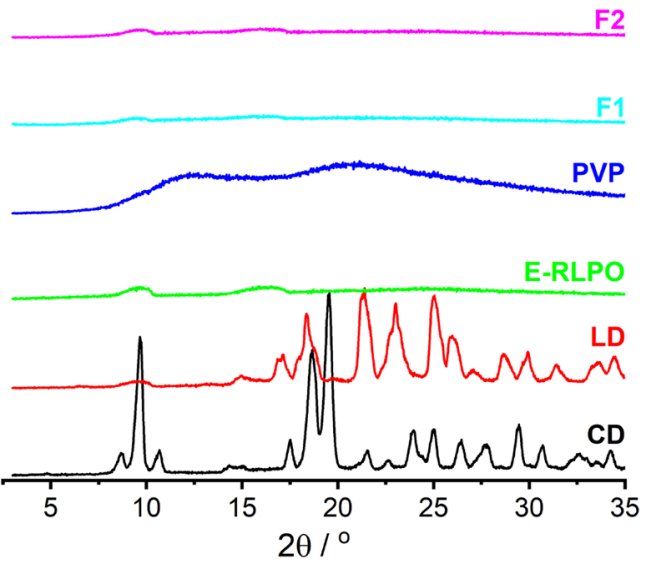

Fig. 4 XRD diffraction patterns of the raw APIs (LD and CD); the pure polymers (PVP and E-RLPO) and the coaxial fibre formulations (F1 and F2)

at $1626 \mathrm{~cm}^{-1}$. The spectra of the raw polymer powders (Fig. 6b) show that PVP has a broad band between 3400 to $2950 \mathrm{~cm}^{-1}$ corresponding to $\mathrm{O}-\mathrm{H}$ stretches from adsorbed water, $\mathrm{C}=\mathrm{O}$ vibrations at $1654 \mathrm{~cm}^{-1}$, and a $\mathrm{C}-\mathrm{N}$ stretch at $1285 \mathrm{~cm}^{-1}$. The spectrum of E-RLPO displays $\mathrm{C}-\mathrm{H}$ stretching at $2951 \mathrm{~cm}^{-1}$ and $1448 \mathrm{~cm}^{-1}$, a C $=\mathrm{O}$ stretch at $1723 \mathrm{~cm}^{-1}$, and $\mathrm{C}-\mathrm{O}-\mathrm{C}$ vibrations at 1237 and $1143 \mathrm{~cm}^{-1}$.

In the FTIR spectra of the coaxial fiber mats (Fig. 6b), formulation F1 shows E-RLPO bands at $2951 \mathrm{~cm}^{-1}(\mathrm{C}-\mathrm{H}$ stretching), $1728 \mathrm{~cm}^{-1}$ ( $\mathrm{C}=\mathrm{O}$ stretching), and $1148 \mathrm{~cm}^{-1}$ (C-O-C vibrations). Bands from PVP can be seen between 3400 and $2950 \mathrm{~cm}^{-1}$ (O-H and C-H stretches), at $1652 \mathrm{~cm}^{-1}$

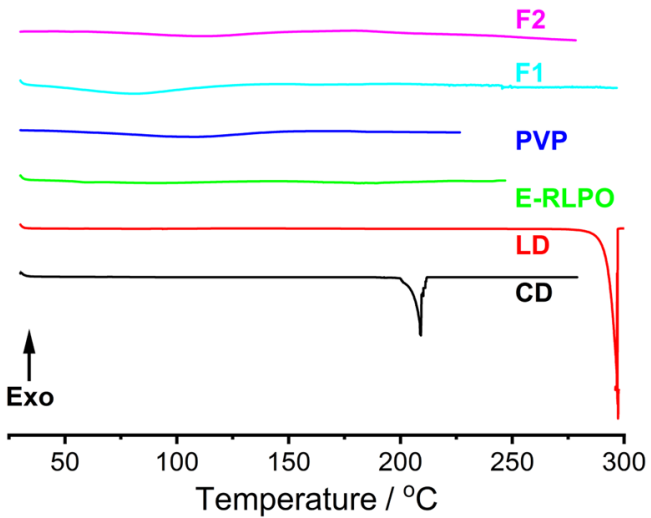

Fig. 5 DSC data for the raw materials and fibers

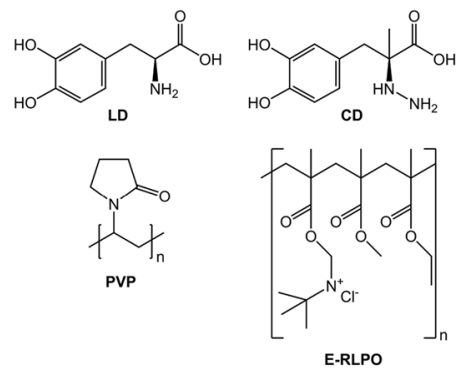

(a)

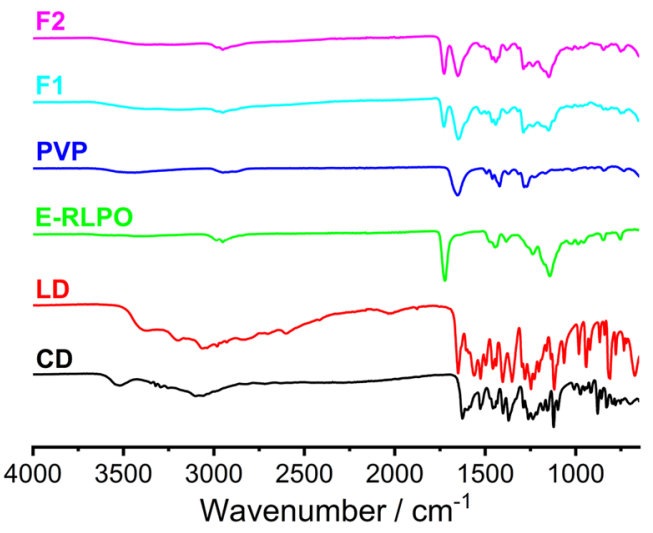

(b)

Fig. 6 a Chemical structures of the APIs and polymers, together with b FTIR spectra

from $\mathrm{C}=\mathrm{O}$ vibrations, and at $1289 \mathrm{~cm}^{-1}$ from $\mathrm{C}-\mathrm{N}$ stretching. Similarly, for formulation F2, peaks from E-RLPO can be seen at $2951 \mathrm{~cm}^{-1}, 1726 \mathrm{~cm}^{-1}$, and at $1150 \mathrm{~cm}^{-1}$, and vibrations from PVP are visible at 3400 to $2984 \mathrm{~cm}^{-1}, 2950 \mathrm{~cm}^{-1}$, $1651 \mathrm{~cm}^{-1}$, and $1289 \mathrm{~cm}^{-1}$. In comparison to the spectra of pure $\mathrm{LD}$ and $\mathrm{CD}$, in the fibers many of the drugs' characteristic peaks have shifted positions or merged with the peaks from the carrier polymers, indicating that the APIs were successfully loaded in the fiber mat with significant intermolecular interactions between the drug and the polymer. 


\section{Drug Loading and Encapsulation Efficiency}

The drug loading and encapsulation efficiency were determined by HPLC, and the results are listed in Table 2. The loadings are in most cases very close to $100 \%$ of the theoretical amount. While the calculated loadings come out at a little over $100 \%$ in places, the error of the measurements is such that these values are essentially identical to the theoretical content. The deviations seem can be ascribed to inhomogeneities in the density of the fiber mat. The exception is for $\mathrm{CD}$ in the $\mathrm{F} 2$ formulation where the encapsulation efficiency (EE) is ca. $80 \%$. This decrease in CD content could be a result of degradation during either the electrospinning or HPLC sample preparation processes. The observation of close to $100 \% \mathrm{EE}$ in most cases supports the DSC data in indicating that there is very little solvent present in the fibers. Given the loadings calculated in Table 2, to deliver a therapeutic dose of $100 \mathrm{mg} \mathrm{LD} / 25 \mathrm{mg}$ $\mathrm{CD}$, around $500 \mathrm{mg}$ of the F1 fibers would be required. This is an eminently feasible size for a dosage form.

\section{Drug Release Studies}

In the British Pharmacopoeia (BP), it is recommended to perform dissolution testing of the $\mathrm{LD} / \mathrm{CD}$ combination SR tablet in $0.1 \mathrm{M} \mathrm{HCl}$ [43]. This protocol was followed here. The SR tablet contains $\mathrm{LD} / \mathrm{CD}$ at $200 / 50 \mathrm{mg}$. This is relatively high, and thus the high drug-loaded formulation F1 seemed more appropriate for study here. Further, the TEM images of F2 did not confirm the formation of core/shell fibers (Fig. 3). The F1 fibers were manually loaded into a hard gelatin capsule (size 0 ) for release studies, since the intention is to deliver the formulation orally. The in vitro drug release results are given in Fig. 7 a.

In Fig. 7a, the release of both drugs from the Sinemet CR tablet show similar trends, with ca. 50\% of the loaded drug released in the first hour, and the remaining 50\% in the second hour. To allow a direct comparison with the SR tablet, drug release from the formulations was explored in $\mathrm{HCl}$. This will allow the drug molecules to be released and dissolve into the acidic medium of the stomach, avoiding degradation. Since the fibers are expected to have mucoadhesive properties, they could be developed into gastro-retentive systems to this end. The in vitro drug release data are presented in Fig. 7a. The release of both drugs was similar, showing a multiphasic release profile. In the first $2 \mathrm{~h}$, over $25 \%$ of the loaded drug

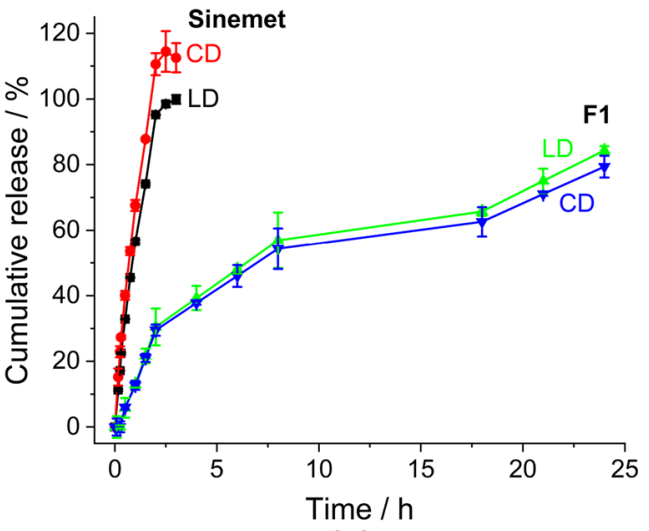

(a)

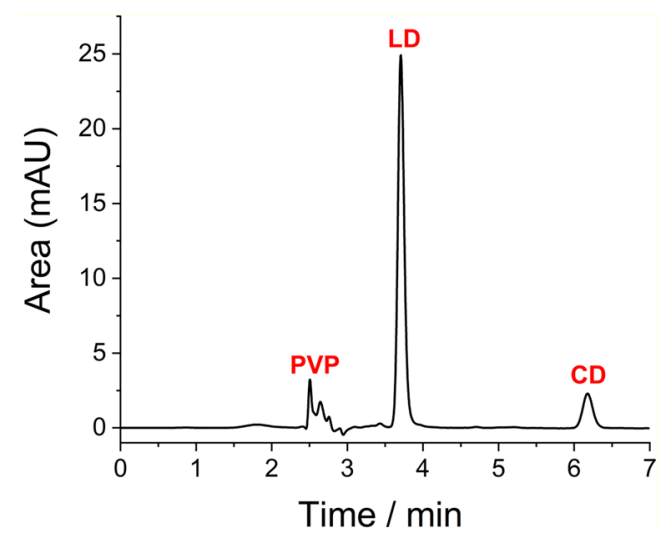

(b)

Fig. 7 a In vitro dissolution profiles showing release of LD and CD from Sinemet $\mathrm{CR}^{\circledR} 200 / 50 \mathrm{mg}$ tablets and $\mathrm{F} 1$ at $\mathrm{pH} 1$. Data are reported as mean \pm S.D. $(n=3)$. b A representative HPLC chromatogram, taken after $24 \mathrm{~h}$ of immersion of $\mathrm{F} 1$ at $\mathrm{pH} 1$

was rapidly released, followed by another $25 \%$ release over the next $6 \mathrm{~h}$. Half the loading of each drug is present with hydrophilic PVP in the shell layer, and it is expected that it is this half of the dose which was freed in the first stages of release. A similar rapid release pattern from PVP has been reported from coaxial fibers made of a PVP shell and ethyl cellulose core [29].

Drug release from the E-RLPO core can be seen to take place over 8 to $24 \mathrm{~h}$. After $24 \mathrm{~h}, 84 \pm 1 \%$ of the LD and $79 \pm 3 \%$ of the CD loading has been freed into solution. There is no evidence for degradation products in the HPLC data (Fig. 7b), and thus the residual $\mathrm{LD}$ and $\mathrm{CD}$ must be retained inside the

Table 2 The drug loadings and EE of the fibers. EE $(\%)=100 \times$ actual loading/theoretical loading. Data are reported as mean \pm S.D. $(n=3)$

\begin{tabular}{llllr}
\hline Formulation & LD loading $(\% \mathrm{w} / \mathrm{w})$ & LD EE $(\%)$ & CD loading $(\%$ w/w) & CD EE $(\%)$ \\
\hline F1 & $19.1 \pm 0.6$ & $100 \pm 3$ & $4.9 \pm 0.1$ & $103 \pm 2$ \\
F2 & $9.4 \pm 0.0$ & $105 \pm 0$ & $1.8 \pm 0.0$ & $83 \pm 1$ \\
\hline
\end{tabular}


fibers even after $24 \mathrm{~h}$. As described earlier, E-RLPO is a waterinsoluble polymer with low permeability, but it undergoes $\mathrm{pH}$-independent swelling allowing it to slowly release a drug cargo [41]. The slow release of LD and CD observed from E-RLPO arises due to diffusion of the loaded drug through and out of the polymer matrix [44]. This extended release from the core layer is a comparable to results reported by Pornsopone et al. who showed E-RLPO fibers loaded with $10 \%$ $\mathrm{w} / \mathrm{w}$ indomethacin to reach only $18 \%$ cumulative release after $24 \mathrm{~h}$ [35]. Overall, it is clear that the F1 formulation provides the desired release profile. The results here are similar to others reported for coaxial fibers, for instance those of Yu et al. where core/shell systems were prepared with the non-steroidal anti-inflammatory agent ketoprofen, PVP as the shell polymer and either zein [32] or ethyl cellulose [29] as the core matrix. Other authors have employed a cellulose acetate shell and a core containing sodium hyaluronate and naproxen-loaded liposomes to achieve a biphasic release profile [45]. In this work however, we make an advance on these previous studies by incorporating multiple active ingredients into each compartment of the fiber architecture.

\section{Conclusions}

This study aimed to employ coaxial electrospinning to fabricate a core/shell fiber fixed dose formulation. Two polymers, PVP and E-RLPO, were used to build the core/shell fibers. In each layer, two model drugs (LD and CD) were loaded, resulting in an FDC which could be used for the treatment of PD. We were able to develop fibers with theoretical drug loadings of LD/CD at 19.0/4.8\% (F1) and 8.9/2.2\% w/w (F2). SEM images showed the production of cylindrical fibers with smooth surfaces. TEM images confirmed the formation of coaxial structure in the F1 fibers, but not for F2. Physical form characterisation studies with XRD and DSC revealed that both formulations comprised amorphous solid dispersions. Drug-polymer interactions between both drugs and the polymers were confirmed with FTIR spectroscopy. Encapsulation efficiencies were close to $100 \%$ in most cases. An in vitro dissolution study revealed the ability of the core/ shell fibers to provide extended release profiles, providing an immediate burst release (giving a loading dose) followed by extended release for longer than was observed with commercial controlled release tablets. The F1 fibers should thus be able to help maintain a constant LD plasma concentration, reducing the dosing frequency and undesirable side effects from overuse of the conventional treatments in PD.

Acknowledgements The authors thank the Umm AlQura University (Kingdom of Saudi Arabia) for provision of a scholarship to HB. We also gratefully acknowledge Dr Andrew Weston and Mrs Kate Keen for assistance with obtaining SEM and TEM images.

\section{Compliance with Ethical Standards}

Conflict of interest The authors declare that they have no conflict of interest.

Open Access This article is licensed under a Creative Commons Attribution 4.0 International License, which permits use, sharing, adaptation, distribution and reproduction in any medium or format, as long as you give appropriate credit to the original author(s) and the source, provide a link to the Creative Commons licence, and indicate if changes were made. The images or other third party material in this article are included in the article's Creative Commons licence, unless indicated otherwise in a credit line to the material. If material is not included in the article's Creative Commons licence and your intended use is not permitted by statutory regulation or exceeds the permitted use, you will need to obtain permission directly from the copyright holder. To view a copy of this licence, visit http://creativecommons.org/licenses/by/4.0/.

\section{References}

1. Grosset D, Taurah L, Burn DJ, MacMahon D, Forbes A, Turner K, et al. A multicentre longitudinal observational study of changes in self reported health status in people with Parkinson's disease left untreated at diagnosis. J Neurol Neurosurg Psychiatry 2007, 78, 465.

2. Leopold NA, Polansky M, Hurka MR. Drug adherence in Parkinson's disease. Mov Disord 2004, 19, 513-7.

3. Kulkarni AS, Balkrishnan R, Anderson RT, Edin HM, Kirsch $\mathrm{J}$, Stacy MA. Medication adherence and associated outcomes in medicare health maintenance organization-enrolled older adults with Parkinson's disease. Mov Disord 2008, 23, 359-65.

4. Fernandez HH. Updates in the medical management of Parkinson disease. Cleve Clin J Med 2012, 79, 28-35.

5. Ngwuluka N, Pillay V, Du Toit LC, Ndesendo V, Choonara Y, Modi $\mathrm{G}$ et al. Levodopa delivery systems: Advancements in delivery of the gold standard. Expert Opin Drug Deliv 2010, 7, 203-24.

6. https://www.nice.org.uk/guidance/ng71/chapter/recommendations

7. Contin M, Riva R, Martinelli P, Procaccianti G, Cortelli P, Avoni $\mathrm{P}$, et al. Response to a standard oral levodopa test in Parkinsonian patients with and without motor fluctuations. Clin Neuropharmacol 1990, 13, 1-28.

8. Seeberger LC, Hauser RA. Optimizing bioavailability in the treatment of Parkinson's disease. Neuropharmacology 2007, 53, 791-800.

9. Grosset D, Antonini A, Canesi M, Pezzoli G, Lees A, Shaw K, et al. Adherence to anti-Parkinson medication in a multicenter European study. Mov Disord 2009, 24, 826-32.

10. Contin M, Martinelli P. Pharmacokinetics of levodopa. J Neurol 2010, 257, S253-61.

11. Hsu A, Yao HM, Gupta S, Modi NB. Comparison of the pharmacokinetics of an oral extended-release capsule formulation of carbidopa-levodopa (Ipx066) with immediate-release carbidopa-levodopa (Sinemet((R))), sustained-release carbidopa-levodopa (Sinemet((R)) CR), and carbidopa-levodopa-entacapone (Stalevo((R))). J Clin Pharmacol 2015, 55, 995-1003.

12. https://www.nice.org.uk/guidance/qs164/chapter/Quality-state ment-4-Levodopa-in-hospital-or-a-care-home. 
13. Stocchi F. The hypothesis of the genesis of motor complications and continuous dopaminergic stimulation in the treatment of Parkinson's disease. Parkinsonism Relat Disord 2009, 15 Suppl 1, S9-S15.

14. Williams GR, Raimi-Abraham BT, Luo CJ. Nanofibres in drug delivery. London: UCL Press; 2018.

15. Yu DG, Li JJ, Williams GR, Zhao M. Electrospun amorphous solid dispersions of poorly water-soluble drugs: A review. J Control Release 2018, 292, 91-110.

16. Yu D-G, Wang M, Li X, Liu X, Zhu L-M. Annie Bligh SW. Multifluid electrospinning for the generation of complex nanostructures. Wiley Interdiscip Rev Nanomed Nanobiotechnol 2019. https ://doi.org/10.1002/wnan.1592.

17. Illangakoon UE, Gill H, Shearman GC, Parhizkar M, Mahalingam S, Chatterton NP et al. Fast dissolving paracetamol/caffeine nanofibers prepared by electrospinning. Int J Pharm 2014, 477, 369-79.

18. Tort S, Yıldız A, Tuğcu-Demiröz F, Akca G, Kuzukıran Ö, Acartürk F. Development and characterization of rapid dissolving ornidazole loaded PVP electrospun fibers. Pharm Dev Tech 2019, 24, 864-73.

19. Giram PS, Shitole A, Nande SS, Sharma N, Garnaik B. Fast dissolving moxifloxacin hydrochloride antibiotic drug from electrospun Eudragit 1-100 nonwoven nanofibrous mats. Mater Sci Eng C 2018, 92, 526-39.

20. Kajdič S, Vrečer F, Kocbek P. Preparation of poloxamer-based nanofibers for enhanced dissolution of carvedilol. Eur J Pharm Sci 2018, 117, 331-40.

21. Feng K, Li C, Wei Y-S, Zong M-H, Wu H, Han S-Y. Development of a polysaccharide based multi-unit nanofiber mat for colon-targeted sustained release of salmon calcitonin. J Colloid Interface Sci 2019, 552, 186-95.

22. Dias AM, da Silva FG, Monteiro APdF, Pinzón-García AD, Sinisterra RD, Cortés ME. Polycaprolactone nanofibers loaded oxytetracycline hydrochloride and zinc oxide for treatment of periodontal disease. Mater Sci Eng C 2019, 103, 109798.

23. Angkawinitwong U, Awwad S, Khaw PT, Brocchini S, Williams GR. Electrospun formulations of bevacizumab for sustained release in the eye. Acta Biomater 2017, 64, 126-36.

24. Jin M, Yu DG, Geraldes CF, Williams GR, Bligh SW. Theranostic fibers for simultaneous imaging and drug delivery. Mol Pharm 2016, 13, 2457-65.

25. Shen X, Yu D, Zhu L, Branford-White C, White K, Chatterton NP. Electrospun diclofenac sodium loaded Eudragit(r) 1 100-55 nanofibers for colon-targeted drug delivery. Int J Pharm 2011, 408, 200-7.

26. Bukhary H, Williams GR, Orlu M. Electrospun fixed dose formulations of amlodipine besylate and valsartan. Int J Pharm 2018, $549,446-55$.

27. Li H, Zhu J, Chen S, Jia L, Ma Y. Fabrication of aqueous-based dual drug loaded silk fibroin electrospun nanofibers embedded with curcumin-loaded RSF nanospheres for drugs controlled release. $R S C A d v \mathbf{2 0 1 7}, 7,56550-8$.

28. Zhang Y, Huang Z-M, Xu X, Lim CT, Ramakrishna S. Preparation of core-shell structured PCL-r-gelatin bi-component nanofibers by coaxial electrospinning. Chem Mater 2004, 16, 3406-9.

29. Yu DG, Wang X, Li XY, Chian W, Li Y, Liao YZ. Electrospun biphasic drug release polyvinylpyrrolidone/ethyl cellulose core/ sheath nanofibers. Acta Biomater 2013, 9, 5665-72.

30. Wang C, Yan K-W, Lin Y-D, Hsieh PCH. Biodegradable core/shell fibers by coaxial electrospinning: Processing, fiber characterization, and its application in sustained drug release. Macromolecules 2010, 43, 6389-97.
31. Chou SF, Carson D, Woodrow KA. Current strategies for sustaining drug release from electrospun nanofibers. J Control Release 2015, 220, 584-91.

32. Jiang YN, Mo HY, Yu DG. Electrospun drug-loaded core-sheath $\mathrm{PVP} /$ zein nanofibers for biphasic drug release. Int J Pharm 2012 , 438, 232-9.

33. Commission BP. British pharmacopoei. Carbidopa assay standard. London: Stationery Office; 2013.

34. Pornsopone V, Supaphol P, Rangkupan R, Tantayanon S. Electrospinning of methacrylate-based copolymers: Effects of solution concentration and applied electrical potential on morphological appearance of as-spun fibers. Polym Eng Sci 2005, 45, 1073-80

35. Pornsopone V, Supaphol P, Rangkupan R, Tantayanon S. Electrospun methacrylate-based copolymer/indomethacin fibers and their release characteristics of indomethacin. J Polym Res 2007, $14,53-9$

36. Illangakoon UE, Yu DG, Ahmad BS, Chatterton NP, Williams GR. 5-fluorouracil loaded Eudragit fibers prepared by electrospinning. Int J Pharm 2015, 495, 895-902.

37. Yang Y-Y, Zhang M, Wang K, Yu D-G. pH-sensitive polymer nanocoating on hydrophilic composites fabricated using modified coaxial electrospraying. Mater Lett 2018, 227, 93-6.

38. O'Neil MJ, Smith A, Heckelman PE, Budavari S. The Merck index-an encyclopedia of chemicals, dugs, and biologicals. Whitehouse Station: Merck and Inc. Co; 2001. p. 4342.

39. https://www.drugbank.ca/drugs/DB00190.

40. http://polymerdatabase.com/polymers/polyvinylpyrrolidone.html.

41. Dave VS, Fahmy RM, Bensley D, Hoag SW. Eudragit((R)) RSPO/ RLPO as rate-controlling matrix-formers via roller compaction: Influence of formulation and process variables on functional attributes of granules and tablets. Drug Dev Ind Pharm 2012, 38, 1240-53.

42. Parikh T, Singh Gupta SS, Meena A, Serajuddin ATM. Investigation of thermal and viscoelastic properties of polymers relevant to hot melt extrusion: iii-Polymethacrylates and polymethacrylic acid based polymers. J Excip Food Chem 2014, 5, 56-64.

43. Commission BP. British pharmacopoeia. Co-careldopa tablets, Appendix XII B. Annex: Recommendations on dissolution testing. London, England: Stationary Office; 2016.

44. Yadav SK, Mishra S, Mishra B. Eudragit-based nanosuspension of poorly water-soluble drug: Formulation and in vitro-in vivo evaluation. AAPS PharmSciTech 2012, 13, 1031-44.

45. Li Z, Kang H, Che N, Liu Z, Li P, Li W et al. Controlled release of liposome-encapsulated naproxen from core-sheath electrospun nanofibers. Carbohydrate Polymers 2014, 111, 18-24.

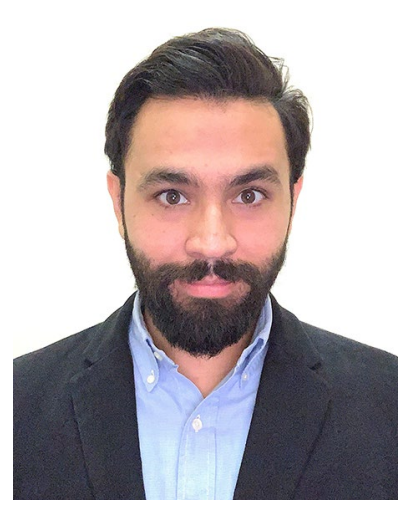

Haitham Bukhary received a PharmD degree from King Abdulaziz University in Saudi Arabia in 2009. He worked as a pharmacist in Saudi Arabia until 2012, when he moved to the UK. He received an MSc in Pharmaceutical Technology from Kings College London in 2014, and then studied for a $\mathrm{PhD}$ in the UCL School of Pharmacy. Upon completion of his $\mathrm{PhD}$ in 2019 , Haitham returned to Saudi Arabia where he is currently Assistant Professor of Pharmaceutics in the College of Pharmacy of

Umm Al-Qura University. 


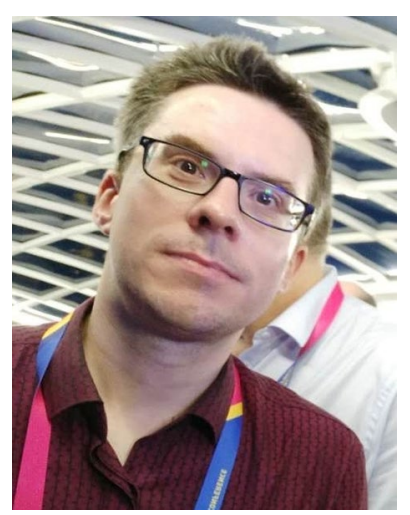

Gareth R. Williams received a MChem (Hons) degree from the University of Oxford in 2002. He remained in Oxford for a DPhil $(\mathrm{PhD})$, which was completed in 2005. In September 2010 he joined London Metropolitan University as a Senior Lecturer in Pharmaceutical Science, and in November 2012 was appointed to the UCL School of Pharmacy as a Lecturer in Pharmaceutics. He was promoted to Associate Professor in 2016 and became Head of Pharmaceutics in 2020. His research interests lie in the development of advanced polymer- and inorganic-based nanoscale drug delivery systems.

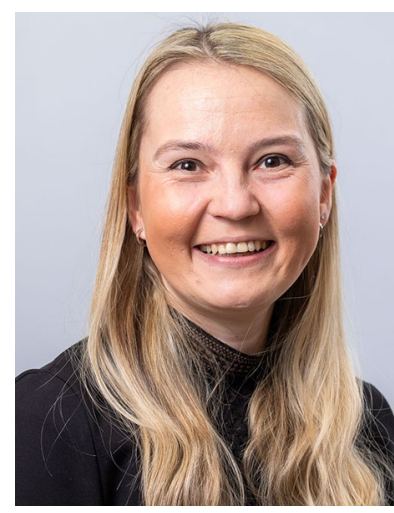

Mine Orlu is an Associate Professor in Pharmaceutics at UCL School of Pharmacy. She prioritizes patient-centric drug delivery by translating high quality basic research into clinical benefit via improved treatments and interventions. Her main research interest is at the interface between clinical pharmacy and pharmaceutics, enabling older patients to have access to safe, effective and acceptable drug delivery systems. 